\title{
Lumbar Trunk Muscle Use in Standing Isometric Heavy Exertions
}

\author{
Albert Schultz, *Ronita Cromwell, David Warwick, and †Gunnar Andersson \\ Department of Mechanical Engineering, University of Michigan, Ann Arbor, Michigan; *Department of Physical \\ Therapy, Chicago Medical School, North Chicago; and †Department of Orthopaedic Surgery, Rush Medical \\ University, Chicago, Illinois, U.S.A.
}

\begin{abstract}
Summary: A study was conducted to see whether a biomechanical model previously validated for predicting the lumbar spine internal loads imposed by the performance of easy and moderately strenuous physical tasks was also adequate for predicting loads imposed by heavy exertions. Lumbar trunk muscle myoelectric activities were measured in 10 healthy young adult men performing a variety of less strenuous and more strenuous tasks while standing upright, and these were compared to the lumbar muscle contraction magnitudes predicted by the model. For the less strenuous tasks, measured activities and predicted forces showed strong linear correlations, confirming the validity of the model at those load levels. Model predictions for the more strenuous tasks were often found to be inadequate. Contrary to model-incorporated assumptions, substantial antagonistic muscle contractions sometimes occurred, intraabdominal pressurization may sometimes have contributed substantially to the maintenance of structural equilibrium, and the ligamentous tissues of the trunk seemed sometimes to develop substantial passive resistances to bending and twisting moments. Key Words: Spine-Biomechanical modelsTrunk muscles-Electromyography.
\end{abstract}

Lumbar trunk muscle contraction forces and lumbar spine loads developed during quasistatic performances of various physical tasks have been reported in a series of studies from the authors' laboratories (1,6-9). In these studies, biomechanical models of the lumbar trunk were used to predict the loads on the trunk internal structures, and experimental measurements of surface myoelectric signal levels and of lumbar intradiscal pressures were used to test the validity of those predictions. The studies initially addressed the performance of tasks that were mechanically simple to analyze and pro-

Address correspondence and reprint requests to Dr. Albert Schultz at Department of Mechanical Engineering, University of Michigan, Ann Arbor, MI 48109-2125, U.S.A. ceeded to more mechanically complex tasks. For the most part, agreement between the forces and loads predicted by the models and those implied by the experimental measurements was good, so that considerable confidence could be placed in the biomechanical models used for force and load prediction.

The present investigation examined model validity under still more stringent conditions. All of the previous studies considered quasistatic tasks that were at most moderately strenuous. This study considered quite strenuous as well as less strenuous quasistatic tasks. The general objective was to determine whether the assumptions used in the biomechanical models, whose validity had been established for easy to moderate physical tasks, were still valid for more strenuous tasks. 


\section{EXPERIMENTAL METHODS}

\section{Subjects and Tasks}

Ten male adult volunteers were studied. Their ages ranged from 19 to 25 years, with a mean of 22 years; heights ranged from 170 to $190 \mathrm{~cm}$, with a mean of $178 \mathrm{~cm}$; and weights ranged from 526 to $824 \mathrm{~N}$, with a mean of $695 \mathrm{~N}$. At the L3 level, their trunk depths ranged from 20 to $28 \mathrm{~cm}$, with a mean of $24 \mathrm{~cm}$, and their trunk widths ranged from 30 to $35 \mathrm{~cm}$, with a mean of $32 \mathrm{~cm}$. They were all in good health, and none had previous back injuries or significant back pain.

The subjects were asked to perform 43 tasks while standing upright and two tasks while lying down (Table 1). The 43 standing tasks were performed in a test cage designed (a) to permit the application of external loads, (b) to provide some control of the subjects' postures, and (c) to enable location of the mass centers of the upper body segments. The subjects were asked to assume comfortable foot and leg positions. Their pelvises were strapped to a support board to stabilize their lower body segments.

Of the 45 tasks, 27 were considered to be "less strenuous." These included 22 load-resisting tasks that involved resisting trunk flexion, extension, lateral bending, and twisting. In these 22 tasks, loads (Table 1) were applied by a system of cords and

TABLE 1. Tasks performed

\begin{tabular}{|c|c|c|c|c|c|c|}
\hline Tasks & \multicolumn{6}{|c|}{ Task sequence No. } \\
\hline \multicolumn{7}{|l|}{ Less strenuous (27) } \\
\hline Stand relaxed & & & & 27 & & \\
\hline Lie supine & & & & 28 & & \\
\hline Lie prone & & & & 29 & & \\
\hline Hold $49 \mathrm{~N}$ at chest & & & & 44 & & \\
\hline Hold $49 \mathrm{~N}$ forward & & & & 45 & & \\
\hline \multicolumn{2}{|l|}{ Resist tasks and loads } & Flx & Ext & RLB & LLB & \\
\hline $40 \mathrm{~N}$ & & 25 & 26 & 42 & 43 & \\
\hline $93 \mathrm{~N}$ & & 19 & 22 & 36 & 39 & \\
\hline $133 \mathrm{~N}$ & & 20 & 23 & 37 & 40 & \\
\hline \multirow[t]{2}{*}{$175 \mathrm{~N}$} & & 21 & 24 & 38 & 41 & \\
\hline & & & RTw & LTw & & · \\
\hline $93 \mathrm{~N}$ & & & 13 & 16 & & \\
\hline $118 \mathrm{~N}$ & & & 14 & 17 & & \\
\hline $158 \mathrm{~N}$ & & & 15 & 18 & & \\
\hline More strenuous (18) & Flx & Ext & RLB & LLB & $\mathrm{RTw}$ & LTw \\
\hline 1/3 MV attempt & 2 & 5 & 31 & 34 & 8 & 11 \\
\hline 2/3 MV attempt & 3 & 6 & 32 & 35 & 9 & 12 \\
\hline MV attempt & 1 & 4 & 30 & 33 & 7 & 10 \\
\hline
\end{tabular}

Flx, flexion; Ext, extension; RLB, right lateral bend; LLB, left lateral bend; RTw, right twist; LTw, left twist; MV, maximum voluntary. pulleys to a harness fastened around the chest just under the arms. Cords were run horizontally from metal rings on the harness over sets of pulleys placed individually at the same heights as the metal rings, and weights were hung from the cords. In the twist-resist tasks, two equal weights were applied to impose pure longitudinal twisting moments. The subjects were asked to remain in the same upright posture and resist the weight applications in different directions. In two other resist tasks, loads were imposed by having the subjects hold weights in both hands. In three other tasks, subjects were asked to stand relaxed, to lie supine, or to lie prone on a cot.

In the 18 "more strenuous" tasks, the chest harness was attached through a cable to a load cell. Subjects were then asked to pull voluntarily against this cable, first with maximum effort, and then with one-third and two-thirds of maximum effort. Load levels were displayed to the subjects for the latter two types of exertions. For voluntary twist tests, exertions were made against padded shoulder bars through a yoke designed to transmit only twisting movements to an overhead torque cell.

Sagittal plane symmetry was maintained both for body configuration in all tasks and for loading in all but the lateral bending and twisting tasks.

\section{Data Measurement}

Two types of measurements were made during task performance. Configuration data were taken to locate both the mass centers of the head, upper limbs, and trunk, and the lines of action and moment arms of the external loads. Myoelectric activities in the trunk muscles were measured with surface electrodes.

\section{Measurement of Configuration Data}

Three targets were fastened to the subject's right side: one at the ear, one at the T9 level, and one at the L3 level. The ear and T9 targets were aligned approximately transverse to the mass centers of the head and the trunk segments superior to L3. The L3 target was aligned approximately transverse to the center of that vertebral body. A horizontal scale was placed on the right side of the test cage. A plumb-bob line slid along the scale and visually aligned with each target was used to estimate the anteroposterior location of each target and the hands to within a few centimeters. In the upright posture 
and for the tasks performed in this study, body segment weight loads usually contribute relatively little to the total imposed moments. Thus, only approximate data on mass center locations were needed. Visual checks were made from the front to assure maintenance of adequate sagittal symmetry of body configuration.

In the load-resist tasks, a tape measure was used to find the vertical distance from the $\mathrm{L} 3$ level to the cord in the flexion, extension, and lateral bending resist tasks, and the distance between the cords in the twisting resist tasks, so that the moments of the external forces at the L 3 vertebral body center could be computed. The other needed moment arms were available from the target data. The width and depth of the trunk at the L3 level were also measured in each subject.

\section{Measurement of Myoelectric Activity}

Myoelectric signals were picked up by eight bipolar recessed surface electrode pairs. Electrode pairs were placed $3 \mathrm{~cm}$ lateral to the midline on both sides of the back at the L3 level. Additional electrode pairs were placed over the rectus abdominus muscles, $2 \mathrm{~cm}$ lateral to the midline on each side at the level of the umbilicus. Electrode pairs were also placed on both sides over the oblique abdominal muscles: medially, at $3 \mathrm{~cm}$ superior and anterior to the iliac spines; and laterally, at the most lateral aspect of the trunk at the same level. The electrodes were filled with electrode jelly and glued to the skin. The longitudinal axis of the electrode pairs was aligned approximately with the direction of the muscle fibers. For the oblique abdominal muscles, alignment was with the direction of the external muscle fibers. The eight channels of myoelectric signals were fed to preamplifiers contained in a lightweight box attached to the test cage. The signals were further amplified in main amplifiers, full-wave rectified, averaged with a time constant of $0.2 \mathrm{~s}$, and recorded on a chart recorder.

\section{Task Performance and Data Evaluation Procedures}

All tasks were performed isometrically. After a 1or 2-s delay to ensure that stable conditions had been achieved, the recording equipment was started and, if called for, configuration measurements were made. Data were taken for $5 \mathrm{~s}$ in the voluntary exertion tasks and for $15 \mathrm{~s}$ in the other tasks, after which the subjects rested for a min- imum of 1 min before performing the next task. Adequate additional rest periods were allowed between groups of tasks. Tasks 1-29 (Table 1) were performed on the first test day, and tasks 30-45 were performed on a second day. There was no subject-reported or EMG signal evidence of fatigue during the tests.

For analysis, the myoelectric signals were examined visually, and any artifacts (those due to a cough or a movement of the arms, for example) were excluded. Averaged values were determined for each subject, channel, and recording period. Means and SD of the averaged values were calculated over the 10 subjects for each of the 45 tasks performed.

\section{BIOMECHANICAL ANALYSES OF TRUNK INTERNAL LOADS}

The contraction forces in the trunk muscles needed to perform a task and the compression and shear loads imposed on the spine by that performance were computed for each subject and each test by biomechanical analyses. Each analysis was based on the requirement that all body segments superior to an imaginary transverse cutting plane at the $\mathrm{L} 3$ level of the spine must remain in equilibrium during task performance. There were two main steps in the analysis. First, the net lower-trunk support reaction needed for equilibrium was computed. Second, the trunk muscle contraction forces that could supply that net reaction and the resulting spine compression and shear loads were estimated.

Computation of the net reaction required knowledge of the magnitudes of the externally applied loads and the weights of all body segments superior to the cutting plane. The externally applied loads were the measured cord-applied or voluntarily developed forces, or the held weights. The weights of the head, each arm, and the trunk above the L3 level were assumed (based on the data of Clauser and co-workers (2) as $5,4.5$, and $36.1 \%$ of the subject's body weight. The points at which these forces and weights were applied were available from the configuration measurements. Once all these data were entered into the six equations expressing the requirements for equilibrium, the six components of the net reaction were computed.

The trunk muscle contraction forces and the spine compression and shear loads were calculated from the net reaction using biomechanical models 
of the L 3 cross-section and solving the model equations by an optimization technique.

The L3 cross-sectional models incorporated 11 bilateral pairs of single muscle equivalents representing the rectus abdominus muscles; the lateral and the medial external oblique abdominal muscles; the lateral and the medial oblique abdominal muscles; the psoas; the quadratus lumborum; the lumbar slips of the latissimus dorsi; and the multifidus, longissimus, and iliocostalis groups of the erector spinae muscles.

The L3 spine motion segment was assumed to resist compression, lateral shear, and anteroposterior shear, but to have no significant moment resistance. Abdominal cavity pressurization was initially not accounted for in the model, because in nonstrenuous isometric performances similar to those in this study, it does not exhibit a consistent relationship with the loads imposed on the spine by task performance, and it provides a mean spine compression relief of only $\sim 15 \%$ (8). Subsequent calculations that accounted for abdominal pressurization are described in the Results section. The L3 cross-sectional geometry was scaled from crosssectional anatomy drawings (4) in proportion to the trunk width and depth of each subject at the L3 level. This scaling was used to locate single equivalent muscle centroids and compute the cross-sectional area for each muscle represented.

The optimization scheme used to calculate internal forces minimized the maximum muscle contraction intensity (stress) required to satisfy the equations of equilibrium. To achieve this, the maximum allowed contraction intensity was, in effect, set to $10 \mathrm{kPa}$, and a solution was sought by linear programming so as to minimize the compression force on the spine. If no solution was available, the allowed intensity was incremented by $10 \mathrm{kPa}$ (minimization was only to within this increment), and a solution was again sought. This intensity incrementation procedure was iterated until a solution was found. Use of this optimization scheme keeps antagonistic muscle activity to a minimum while it calls on nearly every muscle that can contribute to an activity to do so at nearly equal contraction intensities. Full details of the biomechanical analysis techniques are given by Schultz and co-workers (9).

For each of the 45 tasks, the mean and SD over the 10 subjects for each of the calculated internal forces were determined.

\section{Correlation of Prediction and Measurements}

To test the validity of the assumptions made in the biomechanical analyses, the mean measured myoelectric signal amplitudes were correlated with the corresponding mean-predicted muscle contraction forces by a linear least-squares regression analysis, both over all 45 tasks and over only the 27 less strenuous tasks (see Results section). Each of the tasks provided one data point for each correlation.

The predicted contraction forces in the rectus abdominus muscles were correlated on each side with their corresponding myoelectric signal levels, the predicted iliocostalis contraction forces were correlated on each side with the signals detected by the electrodes placed on the back, and the sum of the predicted contraction forces in the internal and external oblique abdominal muscles were correlated on each side with the myoelectric signal levels in the electrodes placed over these muscles for both the medial and the lateral locations.

\section{RESULTS}

\section{External Moments Developed}

The 10 subjects developed mean maximum voluntary exertion moments about the L3 level that ranged from 85 to $205 \mathrm{Nm}$, whereas the moments in the most strenuous resist tests ranged from 37 to 51 $\mathrm{Nm}$ (Table 2). The ratios of maximum resist test moments to maximum voluntary exertion moments were $\sim 20 \%$ in flexion, extension, and lateral bending, $\sim 60 \%$ in twisting. The maximum exertion moments developed during flexion, extension, and lateral bending were quite similar to those reported earlier by McNeill and colleagues (5). Data on maximum moments developed during standing twist tests were not found in the literature.

TABLE 2. Mean moments ( $\mathrm{Nm}$ ) developed in selected tasks

\begin{tabular}{lrrrc}
\hline $\begin{array}{c}\text { Maximum voluntary } \\
\text { attempted exertion }\end{array}$ & \multicolumn{2}{c}{ Largest resist } & $\begin{array}{c}\text { Resist/ } \\
\text { MVE ratio }\end{array}$ \\
\hline Ext & $204(78)$ & 175 N Flx & $43(8)$ & 0.21 \\
Flx & $191(70)$ & 175 N Ext & $37(6)$ & 0.19 \\
RLB & $164(56)$ & 175 N LLB & $39(9)$ & 0.24 \\
LLB & $180(65)$ & 175 N RLB & $39(9)$ & 0.22 \\
RTw & $91(32)$ & 158 N LTw & $51(0)$ & 0.56 \\
LTw & $85(30)$ & 158 N RTw & $51(0)$ & 0.60 \\
\hline
\end{tabular}

Abbreviations as in Table 1.

SD in parentheses. 


\section{Myoelectric Signal Levels}

Mean values of the myoelectric signal levels ranged from 8 to $201 \mu \mathrm{V}$ (Table 3). The largest signal levels in the erector spinae electrodes occurred during maximum voluntary attempted extension. In the rectus abdominus and the medial oblique abdominal electrodes, the largest signal levels occurred during maximum voluntary attempted flexion, but in the lateral oblique abdominal electrodes, they occurred in the ipsilateral electrode upon attempted lateral bending.

Compared with the largest myoelectric signal level recorded by each electrode for any resist task, the largest signal level values for the maximum exertions were 2.72 and 2.81 times larger in the left and right erector spinae electrodes, 3.92 and 3.76 times larger in the rectus abdominus electrodes, 2.58 and 3.86 times larger in the medial oblique abdominal electrodes, and 4.94 and 5.22 times larger in the left and right lateral abdominal oblique electrodes. This implies that the lumbar trunk muscles and thus the lumbar spine motion segments were heavily loaded by the maximum standing exertions studied.

\section{Internal Loads Predicted by the Biomechanical Model}

For the most strenuous exertions, the biomechanical model predicted mean muscle contraction

TABLE 3. Mean measured myoelectric signal levels $(\mu V)$ of selected tasks

\begin{tabular}{|c|c|c|c|c|c|c|c|c|}
\hline \multirow[b]{2}{*}{ Exercise } & \multicolumn{4}{|c|}{ Left side electrodes } & \multicolumn{4}{|c|}{ Right side electrodes } \\
\hline & ES & RA & OM & OL & ES & RA & OM & OL \\
\hline Stand relaxed & 18 & 11 & 12 & 10 & 13 & 10 & 13 & 10 \\
\hline Supine & 17 & 10 & 12 & 10 & 10 & 9 & 11 & 10 \\
\hline Prone & 17 & 10 & 12 & 10 & 10 & 9 & 12 & 9 \\
\hline Ext 1/3MVS & 71 & 13 & 18 & 15 & 67 & 11 & 21 & 14 \\
\hline Ext 2/3MVS & 133 & 14 & 29 & 25 & 122 & 14 & 35 & 21 \\
\hline Ext MVS & 201 & 21 & 47 & 34 & 189 & 18 & 55 & 29 \\
\hline 175 N Flx Res & 70 & 11 & 12 & 11 & 58 & 9 & 12 & 11 \\
\hline $49 \mathrm{~N}$ Hold out & 82 & 11 & 14 & 11 & 74 & 10 & 13 & 11 \\
\hline Flx 1/3MVS & 13 & 37 & 36 & 15 & 10 & 31 & 38 & 18 \\
\hline Flx $2 / 3 \mathrm{MVS}$ & 13 & 106 & 81 & 30 & 11 & 108 & 84 & 40 \\
\hline Flx MVS & 17 & 165 & 120 & 55 & 18 & 162 & 140 & 58 \\
\hline 175 N Ext Res & 17 & 49 & 54 & 21 & 12 & 49 & 44 & 22 \\
\hline RLB $1 / 3 \mathrm{MVS}$ & 15 & 15 & 14 & 11 & 44 & 20 & 40 & 31 \\
\hline RLB $2 / 3 \mathrm{MVS}$ & 16 & 26 & 25 & 14 & 73 & 46 & 83 & 61 \\
\hline RLB MVS & 26 & 32 & 36 & 18 & 124 & 89 & 129 & 119 \\
\hline 175 N LL Res & 16 & 11 & 15 & 11 & 49 & 24 & 40 & 30 \\
\hline RTw 1/3MVS & 28 & 10 & 31 & 17 & 22 & 11 & 25 & 17 \\
\hline RTw $2 / 3$ MVS & 40 & 20 & 63 & 30 & 37 & 21 & 66 & 36 \\
\hline RTw MVS & 48 & 23 & 106 & 46 & 42 & 28 & 100 & 52 \\
\hline 158 N LTw Res & 23 & 10 & 28 & 15 & 22 & 9 & 20 & 14 \\
\hline
\end{tabular}

ES, erector spinae; RA, rectus abdominus; OM, medial oblique abdominals; OL, lateral oblique abdominals; other abbreviations as in Table 1. forces of $674 \mathrm{~N}$ per side both in the longissimus and in the iliocostalis, $315 \mathrm{~N}$ in the rectus abdominus, $829 \mathrm{~N}$ in the medial oblique abdominal and $770 \mathrm{~N}$ in the lateral oblique abdominal muscles (Table 4). The largest predicted mean muscle contraction intensities needed to equilibrate the external loads were $-300-400 \mathrm{kPa}$ for maximum voluntary attempted lateral bending and twisting, and $\sim 600-700 \mathrm{kPa}$ for attempted flexion and extension. The largest predicted mean L3 motion segment compression was $5,318 \mathrm{~N}$ and occurred in the attempted extension task. The largest mean L3 motion segment shears were $601 \mathrm{~N}$ anteroposteriorly and $375 \mathrm{~N}$ laterally.

We will subsequently argue that these quoted maximum magnitudes are unrealistically high. Major assumptions made in constructing the biomechanical model, although valid for the less strenuous exertions, appear invalid for the more strenuous exertions examined in this study. Thus, the Table 4 entries are probably realistic for the less strenuous tasks, but not for the more strenuous tasks, as are the values of the maximum motion segment loads and muscle contraction intensities just quoted.

\section{Correlations Between Model-Predicted Muscle Contraction Forces and Myoelectric Signal Levels}

Linear regressions were made between the mean predicted muscle contraction forces and the mean myoelectric signal levels for each of the eight electrodes pairs over the 27 less strenuous tasks. The coefficients of linear correlation obtained were 0.85 and 0.74 in the left and right lateral oblique abdominals and exceeded 0.90 at each of the other six electrode sites (Table 5). Thus, for all the less strenuous tasks, a single regression line strongly correlated the model-predicted muscle contraction forces and the measured myoelectric signal levels for each electrode pair.

For all eight electrode pairs and in almost all the types of tasks performed, the relation between the model-predicted muscle contraction forces and the measured myoelectric signal levels for each type of task (attempted extensions, extension rèsists, etc.) was nearly linear (Fig. 1 presents sample results). The slope of the lines was found to vary, sometimes considerably, among types of tasks, however. 
TABLE 4. Mean muscle contraction forces computed by the biomechanical model, $N$ : selected tasks

\begin{tabular}{|c|c|c|c|c|c|c|c|c|c|c|}
\hline \multirow[b]{2}{*}{ Exercise } & \multicolumn{5}{|c|}{ Left side muscles } & \multicolumn{5}{|c|}{ Right side muscles } \\
\hline & ESL & ESI & RA & OM & $\mathrm{OL}$ & ESL & ESI & $\mathrm{RA}$ & OM & OL \\
\hline Stand relaxed & - & - & 8 & 19 & - & - & - & 8 & 19 & - \\
\hline Supine & - & - & - & - & - & - & - & - & - & - \\
\hline Prone & - & - & - & - & - & - & - & - & - & - \\
\hline Ext 1/3MVS & 220 & 219 & - & - & - & 220 & 219 & - & - & - \\
\hline Ext 2/3MVS & 443 & 443 & - & - & - & 443 & 443 & - & - & 一 \\
\hline Ext MVS & 674 & 674 & - & - & - & 674 & 674 & - & - & - \\
\hline 175 N Flx Res & 135 & 135 & - & - & - & 135 & 135 & - & - & - \\
\hline $49 \mathrm{~N}$ Hold out & 136 & 136 & - & - & - & 136 & 136 & - & - & - \\
\hline Flx 1/3MVS & - & - & 111 & 293 & 244 & - & - & 111 & 293 & 244 \\
\hline Flx $2 / 3 \mathrm{MVS}$ & - & - & 214 & 565 & 497 & - & - & 214 & 565 & 497 \\
\hline Flx MVS & - & - & 315 & 829 & 770 & - & - & 315 & 829 & 770 \\
\hline 175 N Ext Res & - & - & 69 & 182 & 133 & - & - & 69 & 182 & 133 \\
\hline RLB 1/3MVS & - & - & 5 & 8 & - & 96 & 149 & 55 & 143 & 148 \\
\hline RLB 2/3MVS & - & - & 1 & 14 & - & 249 & 289 & 110 & 279 & 284 \\
\hline RLB MVS & - & - & 2 & 20 & - & 406 & 427 & 163 & 413 & 420 \\
\hline 175 N LL Res & - & - & 6 & 7 & - & 50 & 110 & 38 & 105 & 111 \\
\hline RTw 1/3MVS & 9 & 10 & 2 & 68 & 68 & - & - & - & 64 & 77 \\
\hline RTw $2 / 3$ MVS & 60 & 15 & - & 137 & 137 & - & - & - & 142 & 155 \\
\hline RTw MVS & 109 & 9 & - & 193 & 193 & - & - & - & 206 & 220 \\
\hline 158 N LTw Res & 35 & 18 & - & 109 & 109 & - & - & - & 112 & 124 \\
\hline
\end{tabular}

ESL, Longissimus; ESI, iliocostalis; other abbreviations as in Table 1. Dashes indicate zero entries.

\section{Muscle Contraction Forces Scaled From Myoelectric Signal Levels}

Because the linear correlations between the mean predicted muscle contraction forces and the mean measured myoelectric signal levels over the 27 less strenuous tasks were strong, we extrapolated those linear relationships to estimate what muscle contraction forces were actually used to perform the more strenuous tasks. This extrapolation was based on two assumptions: (a) that the muscle contraction forces predicted by the biome-

TABLE 5. Coefficients of linear correlation between predicted forces and measured myoelectric signal levels: less strenuous tasks

\begin{tabular}{|c|c|}
\hline Muscle group & Correlation coefficient \\
\hline Rectus abdominus, left & 0.97 \\
\hline right & 0.97 \\
\hline Erector spinae, left & 0.98 \\
\hline right & 0.95 \\
\hline Medial oblique abdominals, left & 0.93 \\
\hline right & 0.91 \\
\hline Lateral oblique abdominals, left & 0.85 \\
\hline right & 0.74 \\
\hline
\end{tabular}

The oblique abdominal muscle electrode signals are correlated with the sum of the forces predicted for the internal and external oblique muscles, and the erector spinae electrode signals are correlated with the forces predicted for the iliocostalis group of the erector spinae. chanical model for the less strenuous tasks were reasonably correct; and (b) that the measured myoelectric signal level at each electrode, for both less strenuous and more strenuous tasks, related linearly to the actual contraction forces in the relevant muscles.

The mean myoelectric data were normalized to the largest signal levels produced in the less strenuous tasks. These occurred in (a) the hold-weightsout task for the longissimus and iliocostalis muscles, (b) the $175 \mathrm{~N}$ extension-resist task for the rectus abdominus and medial oblique abdominal muscles, and (c) the contralateral $175 \mathrm{~N}$ bendingresist task for the lateral oblique abdominal muscles. Then, scaling linearly from the predicted contraction forces for those tasks, a set of estimated muscle contraction forces used in the more strenuous tasks was obtained (Table 6).

Based on the above assumptions, the estimated maximum mean muscle contraction forces used in the more strenuous exertions were 370 and $383 \mathrm{~N}$ in the left and right iliocostalis, 271 and $260 \mathrm{~N}$ in the rectus abdominus, 470 and $703 \mathrm{~N}$ in the medial oblique abdominal, and 542 and $579 \mathrm{~N}$ in the left and right lateral oblique abdominal muscles. Thus, these scaled force magnitudes are lower, sometimes considerably lower, than the biomechanical modelpredicted contraction forces. 


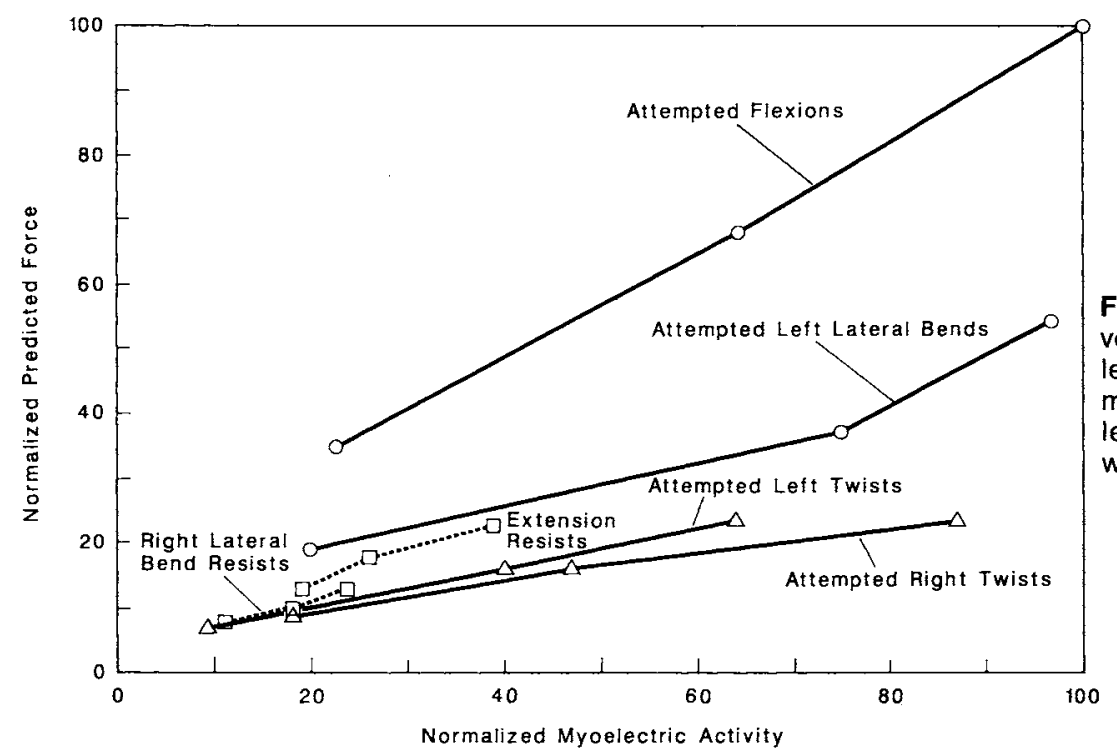

FIG. 1. Model-predicted contraction forces versus measured myoelectric activities in the left medial oblique abdominal muscles. Normalizations are to the largest force or signal level. Approximate linearity is maintained within each type of task.

Differences Between Mean Model-Predicted and Myoelectric-Data-Scaled Muscle Contraction Forces

The biomechanical model used to predict the muscle contraction forces insures that every set of predicted muscle forces satisfies the requirements for mechanical equilibrium. The biomechanical model incorporates three major assumptions: (a)

TABLE 6. Muscle contraction forces scaled from myoelectric data, $N$ : selected tasks

\begin{tabular}{|c|c|c|c|c|c|c|c|c|}
\hline \multirow[b]{2}{*}{ Exercise } & \multicolumn{4}{|c|}{ Left side muscles } & \multicolumn{4}{|c|}{ Right side muscles } \\
\hline & ESI & RA & OM & OL & ESI & RA & OM & OL \\
\hline Stand relaxed & 9 & 3 & 3 & 4 & 6 & 4 & 10 & 6 \\
\hline Supine & 9 & 1 & 2 & - & - & 1 & - & 1 \\
\hline Prone & 8 & 1 & 2 & - & - & 1 & 4 & - \\
\hline Ext 1/3MVS & 114 & 6 & 26 & 35 & 123 & 5 & 52 & 26 \\
\hline Ext 2/3MVs & 237 & 8 & 76 & 99 & 239 & 9 & 130 & 60 \\
\hline Ext MVS & 370 & 19 & 154 & 162 & 383 & 17 & 243 & 105 \\
\hline 175 N Flx Res & 113 & 2 & 1 & 9 & 103 & 1 & 6 & 7 \\
\hline $49 \mathrm{~N}$ Hold out & 137 & 2 & 9 & 8 & 136 & 4 & 9 & 8 \\
\hline Flx $1 / 3 \mathrm{~N}$ & 1 & 48 & 107 & 38 & - & 39 & 145 & 45 \\
\hline Flx $2 / 3$ MVS & - & 168 & 302 & 136 & 3 & 168 & 401 & 162 \\
\hline Flex MVS & 9 & 271 & 470 & 296 & 17 & 260 & 703 & 255 \\
\hline 175 N Ext Res & 8 & 69 & 182 & 72 & 4 & 69 & 182 & 67 \\
\hline RLB 1/3MVS & 4 & 9 & 9 & 8 & 73 & 20 & 160 & 115 \\
\hline RLB 2/3MVS & 6 & 29 & 56 & 28 & 134 & 65 & 395 & 272 \\
\hline RLB MVS & 25 & 39 & 107 & 53 & 243 & 137 & 645 & 579 \\
\hline 175 N LL Res & 7 & 2 & 15 & 6 & 84 & 27 & 160 & 111 \\
\hline RTw 1/3MVS & 31 & 1 & 85 & 47 & 27 & 5 & 79 & 40 \\
\hline RTw $2 / 3 \mathrm{MVS}$ & 53 & 18 & 221 & 137 & 58 & 21 & 303 & 143 \\
\hline RTw MVS & 69 & 24 & 411 & 238 & 69 & 33 & 484 & 224 \\
\hline 158 N LTw R & 20 & - & 71 & 37 & 25 & 1 & 46 & 21 \\
\hline
\end{tabular}

The Results section explains how these scaled forces were obtained. Abbreviations as in Tables 1, 3, and 4.
Antagonistic muscle contractions are minimal; (b) the ligamentous tissues, in particular those of the L3 motion segment, provide negligible passive resistances to the applied moments; and (c) intraabdominal cavity pressurization has negligible effects in quasistaticly performed tests.

All assumptions seemed to be experimentally validated by the less strenuous exertion data. It is difficult to explain otherwise the high degree of linear correlation between model-predicted contraction forces and measured myoelectric signal levels.

To examine whether the three assumptions were valid for the more strenuous exertions, we subtracted the mean myoelectric-data-scaled force from the mean model-predicted force and expressed the difference as a percentage of the largest scaled mean force in that muscle. We used the results of this calculation (Table 7) to examine the validity of the three assumptions in question when the tasks were strenuous.

Before we describe the results, the assumption that antagonistic contractions are minimal will be explained more fully. For a relevant example, in attempted right lateral bending, the biomechanical model predicts (Table 4) that the medial oblique abdominal muscles on the left side will be contracted by a small amount. In one sense, these contractions are antagonistic in that they produce a left lateral bending moment, whereas the "agonists" all contribute to producing the needed right lateral bending movement. The left side contractions are needed for mechanical equilibrium, however. The 
TABLE 7. Model-predicted forces minus scaled forces, as percentage of maximum scaled force

\begin{tabular}{|c|c|c|c|c|c|c|c|c|}
\hline \multirow[b]{2}{*}{ Exercise } & \multicolumn{4}{|c|}{ Left side muscles } & \multicolumn{4}{|c|}{ Right side muscles } \\
\hline & ESI & RA & $\mathrm{OM}$ & $\mathrm{OL}$ & ESI & $\mathrm{RA}$ & $\mathrm{OM}$ & $\mathrm{OL}$ \\
\hline Rel stand & - & - & - & 一 & - & - & 一 & - \\
\hline Supine & - & - & - & -. & -. & - & - & - \\
\hline Prone & - & - & - & - & - & - & - & - \\
\hline Ext 1/3MVS & 28 & - & - & - & 25 & - & - & - \\
\hline Ext 2/3MVS & 56 & - & -16 & -18 & 53 & - & -19 & -10 \\
\hline Ext MVS & 82 & - & -33 & -30 & 76 & - & -35 & -18 \\
\hline 175 N Flx Res & - & - & - & - & - & - & - & - \\
\hline $49 \mathrm{~N}$ Hold Out & - & - & - & - & - & $\cdots$ & - & - \\
\hline Flx 1/3MVS & - & 23 & 40 & 38 & - & 28 & 21 & 35 \\
\hline Flx 2/3MVS & - & 17 & 56 & 67 & - & 18 & 23 & 58 \\
\hline Flx MVS & - & 16 & 76 & 87 & - & 21 & 18 & 89 \\
\hline $175 \mathrm{~N}$ Ext Res & - & - & - & 11 & - & - & - & 11 \\
\hline RLB $1 / 3$ MVS & - & - & - & - & 20 & 14 & - & - \\
\hline RLB 2/3MVS & - & -10 & - & - & 40 & 18 & -16 & - \\
\hline RLB MVS & - & -13 & -18 & -10 & 48 & 10 & -33 & -27 \\
\hline 175 N LL Res & - & - & - & - & - & - & - & - \\
\hline RTw 1/3MVS & - & - & - & - & - & - & - & - \\
\hline RTw 2/3MVS & -11 & - & -18 & - & -15 & - & -23 & - \\
\hline RTw MVS & -16 & - & -46 & - & -18 & -13 & -40 & - \\
\hline 158 N LT Res & - & - & - & 13 & - & - & - & 18 \\
\hline
\end{tabular}

Differences $<10 \%$ not shown. Positive entries indicate that muscles were contracted less strongly than predicted by the model; negative entries indicate that muscles were contracted more strongly than predicted. Abbreviations as in Tables 1, 3, and 4.

required net lateral bending moment must be developed, but at the same time, the flexion-extension and twisting moments must be kept zero. Left side muscles are contracted so that all of the requirements for equilibrium are met, not just those concerning the lateral bending moment. Whether the left side muscles here are "antagonists" is a matter of definition. In examining the first assumption, our concern is not whether antagonistic contractions occur, but whether they are used minimally, to meet no more than the requirements for mechanical equilibrium.

In some of our strenuous task responses, antagonistic contractions were clearly more than minimal. The myoelectric-data-scaled forces exceeded the model predicted forces, resulting in the negative values in the Table 7 entries. Negative values of at least $25 \%$ of the maximum scaled force magnitudes were found in the oblique abdominal muscles in the maximum attempted extension task, in the maximum attempted right lateral bending task, and in both maximum attempted twist tasks. Negative values were also found in the erector spinae muscles in the maximum attempted left twist task. Nonminimal antagonistic contractions were found in other tasks as well, but in smaller amounts. Thus, the first model assumption seems to have been violated during performance of many of the strenuous tasks.
The second and third assumptions of the model are that the ligamentous tissues of the lumbar trunk provide negligible passive resistances to the applied moments and that intraabdominal pressure has negligible effects. At least one of these two assumptions was also clearly violated during the performance of some of the more strenuous tasks. This is indicated by positive values in the Table 7 entries. Those values occur when the scaled forces are less than the model-predicted forces. Positive values of at least $25 \%$ of the maximum scaled force magnitudes were found in the erector spinae muscles in both the attempted extension and the attempted lateral bending tasks, in which values were as much as $82 \%$, and in the oblique abdominal muscles during the attempted flexion tasks, in which values were as much as $89 \%$. An isolated value exceeded $25 \%$ in the right rectus abdominus muscle. No value $>18 \%$ was found for any of the less strenuous tasks.

When antagonistic contractions are more than minimal, contraction forces larger than predicted would be expected to arise in the agonists to equilibrate the excessive antagonistic contractions. This would result in negative Table 7 entries for the agonists as well. The opposite occurred, however. The agonist contraction forces, rather than being larger than predicted, were much smaller than predicted in the tasks just listed. Given the minimum contraction intensity criterion for optimization and no significant intraabdominal pressure, the model-predicted forces are the smallest forces that can satisfy the requirements both for mechanical equilibrium and minimal antagonistic contractions. Despite this, the myoelectric-data-scaled forces were often considerably smaller than these model-predicted forces, even when substantial nonminimal antagonistic contractions occurred. These results suggest strongly either that in the more strenuous tasks the ligamentous tissues of the lumbar trunk and spine provided a considerable amount of passive moment resistance, that intraabdominal pressurization was significantly affecting lumbar trunk mechanics, or both, contrary to the assumptions made in the biomechnical model about these factors.

\section{Intraabdominal Pressurization and Passive Tissue Resistance Effects}

We found two types of discrepancies between model-predicted and experimentally implied muscle contraction forces in the more strenuous 
exertions: one, when scaled forces well exceeded predicted forces, and the other when the opposite occurred. The first type of discrepancy is reasonably explained by the existence of nonminimal antagonistic contractions. Two possible explanations for the second type of discrepancy involve intraabdominal pressurization and the development of passive moment resistances. In this subsection, we will argue that the second type of discrepancy can be explained only in part by intraabdominal pressurization, and thus the model assumption that ligamentous tissues provide negligible passive resistance is invalid in at least some strenuous tasks. The specific discrepancies of concern here are significantly lower than predicted contraction forces in the erector spinae muscles during attempted extension and attempted lateral bending, and in the oblique abdominal muscles during attempted flexion.

We were unable to measure intraabdominal pressures in our subjects. Those pressures have been measured in other investigations, however. The pressures reported by Eie and Wehn (3) are among the largest in the literature. Even their ordinary study subjects seldom could develop sustained abdominal pressures exceeding $100 \mathrm{~mm} \mathrm{Hg} \mathrm{(13.3}$ $\mathrm{kPa}$ ), but one strong weightlifter was reported able to develop a sustained pressure in excess of 200 $\mathrm{mm} \mathrm{Hg}(26.7 \mathrm{kPa})$.

The scaled cross-sectional anatomy data used in the biomechanical simulation model incorporated an abdominal cavity area of 0.465 times the product of umbilical level trunk width and depth, whose centroid lay anterior to the L3 disc center by 0.24 times trunk depth. Using these data, we calculated the effects of 100 and $200 \mathrm{~mm} \mathrm{Hg}$ abdominal pressures in relieving maximum voluntary exertion loads.

These calculations showed that intraabdominal pressurization had no effect on attempted trunk flexion, since it can develop only an extension moment. Thus, abdominal pressurization does not explain any of the discrepancy in the oblique abdominal muscles. As to the erector spinae muscles, in attempted trunk lateral bending, the mechanical situation is complicated. The calculations described, and others, showed that under at least some circumstances, pressurization might explain that discrepancy. But in attempted trunk extension, even at $200 \mathrm{~mm} \mathrm{Hg}$, abdominal pressure could reduce the needed erector contractions by only $18 \%$, whereas the experimentally implied reduction from model-predicted values was on the order of $40 \%$. Thus, intraabdominal pressurization probably can explain only about one-fourth (since $100 \mathrm{~mm} \mathrm{Hg}$ is a more reasonable pressure level), and at most onehalf, of the magnitude of the discrepancy.

These results seem to imply that in at least some of the strenuous tasks studied, the ligamentous tissues of the lumbar spine passively supply significant resistance to external moments, even in fully upright positions of the trunk. The calculations suggest that passive resistance moments in excess of $50 \mathrm{Nm}$ may sometimes develop.

\section{Data Variation}

The myoelectric activity data showed SDs across the 10-subject population that most often were of the order of one-third the mean signal level, and sometimes larger. The variation of the predicted force data was similar in character to that of the myoelectric activity data. Complete tabulations of all experimental data, all force predictions, and the correlations between them are available from the authors.

\section{DISCUSSION}

Particular caution is needed when interpreting our findings concerning the actions of the oblique abdominal muscles. Myoelectric activities were measured using surface electrodes, which pick up from both sets of muscles and perhaps from the transverse abdominals as well, and correlated with the sum of the model-predicted contraction forces for the external and internal oblique muscles. Over the less strenuous tasks, this procedure yielded a larger coefficient of linear correlation than correlating the activity signals with forces in either the internal or external muscles alone. We were unable to collect data that could provide a more substantial justification for this procedure. The issue is probably not very important in lateral bending exertions, because the model predicts that contractions of both internal and external obliques on one side are required and neither is required on the opposite side. Thus, model predictions can be confirmed using this task because myoelectric activity is large on one side and small on the opposite. In twisting exertions, that is not the case. Contractions of the internal obliques alone on one side and of the external obliques alone on the opposite side are required. Thus, substantial myoelectric activity 
arises on both sides, and the model prediction that one of the two obliques on each side is contracting little cannot be experimentally verified with the procedures we used.

Our findings suggest two avenues for further research. First, it would be useful to know if lumbar motion segments can develop significant moment resistances when they are compressed heavily, perhaps by 2,500 N, but not otherwise deformed. Relevant data are not currently available. Second, objective functions for use in the model calculations need to be devised that can adequately predict the muscle contraction patterns actually employed in the performance of heavy exertions.

\section{CONCLUSIONS}

In upright standing maximum voluntary exertions, 10 healthy, young adult male subjects developed lumbar trunk movements of $\sim 200 \mathrm{Nm}$ during attempted extension, $\sim 190 \mathrm{Nm}$ during attempted flexion, $\sim 170 \mathrm{Nm}$ during attempted lateral bending, and $\sim 90 \mathrm{Nm}$ during attempted twisting.

In each given type of task (voluntary exertions in attempted extension, for example), approximate linearity between mean measured myoelectric signal levels and mean model-predicted muscle contraction forces was maintained for all degrees of exertion.

In less strenuous exertions, mean measured myoelectric signal levels and mean model-predicted muscle contraction forces showed strong linear correlations over all tasks, tending to confirm the assumptions used to make the model predictions and to reaffirm the model validity established in earlier studies. In more strenuous exertions, three key assumptions used to make the model predictions seemed at least sometimes to be violated: (a) During some strenuous exertions, substantial nonminimal antagonistic muscle contractions developed; (b) during some strenuous exertions, the results suggested that the ligamentous tissues of the lumbar trunk passively developed substantial moment resistances; (c) during some strenuous exertions, intraabdominal pressurization effects may have contributed substantially to the maintenance of structural equilibrium, but at best could explain only a part of the differences between model predictions and measurements.

Extrapolating the less strenuous task predicted force/measured myoelectric signal regression lines, then corresponding to the more strenuous task signal levels, the mean maximum muscle contraction forces used to perform the strenuous tasks were $\sim 380 \mathrm{~N}$ on each side in the iliocostalis, $\sim 270$ $\mathrm{N}$ in the rectus abdominus, and $\sim 560 \mathrm{~N}$ in the lateral oblique abdominal muscles. They were $\sim 470$ and $\sim 700 \mathrm{~N}$ in the left and right medial oblique abdominal muscles.

Acknowledgment: The support of Public Health Service Grants NS 20536 and OH 01962 and of the Vennema Endowment for this research is gratefully acknowledged.

\section{REFERENCES}

1. Andersson GBJ, Ortengren R, Schultz AB: Analysis and measurement of the loads on the lumbar spine during work at a table. J Biomech 13:513-520, 1980

2. Clauser D, McConville J, Young J: Weight Volume and Center of Mass of Segments of the Human Body. AMRLTR-69-70, Aerospace Medical Research Laboratory (USAF), Wright-Patterson Air Force Base, Ohio, 1969

3. Eie N, Wehn P: Measurements of the intra-abdominal pressure in relation to weight bearing of the lumbo-sacral spine J Oslo City Hosp 12:205-217, 1962

4. Eycleshymer AC, Schoemaker DM: A Cross-Section Anatomy. Appleton-Century Crofts. New York, 1911

5. McNeill T, Warwick D, Andersson G, Schultz A: Trunk strengths in attempted flexion, extension, and lateral bending in healthy subjects and patients with low-back disorders. Spine 5:529-538, 1980

6. Schultz A, Andersson G, Haderspeck K, Ortengren R, Nordin M, Bjork R: Analysis and measurement of lumbar trunk loads in tasks involving bends and twists. $J$ Biomech $15: 669-675,1982$

7. Schultz A, Andersson G, Ortengren R, Bjork R, Nordin M: Analysis and quantitative myoelectric measurement of loads on the lumbar spine when holding weights in standing postures. Spine 7:390-397, 1982

8. Schultz A, Andersson G, Ortengren R, Haderspeck K, Nachemson A: Loads on the lumbar spine: Validation of a biomechanical analysis by measurements of intradiscal pressures and myoelectric signals. J Bone Joint Surg 64A:713720,1962

9. Schultz A, Haderspeck K, Warwick D, Portillo D: Use of lumbar trunk muscles in isometric performance of mechanically complex standing tasks. J Orthop Res 1:77-91, 1983 